\title{
Adaptive Clause Weight Redistribution
}

\author{
Abdelraouf Ishtaiwi ${ }^{1,2}$, John Thornton ${ }^{1,2}$, Anbulagan $^{3}$, Abdul Sattar ${ }^{1,2}$, and \\ Duc Nghia Pham ${ }^{1,2}$ \\ 1 IIIS, Griffith University, QLD, Australia \\ 2 DisPRR, National ICT Australia Ltd, QLD, Australia \\ 3 Logic and Computation Program, National ICT Australia Ltd, Canberra, Australia \\ \{a.ishtaiwi, j. thornton, anbulagan, abdul. sattar, duc-nghia.pham\}@nicta.com. au
}

\begin{abstract}
In recent years, dynamic local search (DLS) clause weighting algorithms have emerged as the local search state-of-the-art for solving propositional satisfiability problems. However, most DLS algorithms require the tuning of domain dependent parameters before their performance becomes competitive. If manual parameter tuning is impractical then various mechanisms have been developed that can automatically adjust a parameter value during the search. To date, the most effective adaptive clause weighting algorithm is RSAPS. However, RSAPS is unable to convincingly outperform the best non-weighting adaptive algorithm AdaptNovelty ${ }^{+}$, even though manually tuned clause weighting algorithms can routinely outperform the Novelty ${ }^{+}$heuristic on which AdaptNovelty ${ }^{+}$is based.

In this study we introduce $\mathrm{R}+\mathrm{DDFW}^{+}$, an enhanced version of the DDFW clause weighting algorithm developed in 2005, that not only adapts the total amount of weight according to the degree of stagnation in the search, but also incorporates the latest resolution-based preprocessing approach used by the winner of the 2005 SAT competition $\left(\mathrm{R}+\right.$ AdaptNovelty $\left.^{+}\right)$. In an empirical study we show $\mathrm{R}+\mathrm{DDFW}^{+}$improves on DDFW and outperforms the other leading adaptive $(\mathrm{R}+\mathrm{Adapt}-$ Novelty $\left.^{+}, \mathrm{R}+\mathrm{RSAPS}\right)$ and non-adaptive $\left(\mathrm{R}+\mathrm{G}^{2} \mathrm{WSAT}\right)$ local search solvers over a range of random and structured benchmark problems.
\end{abstract}

\section{Introduction}

Since the development of the Breakout heuristic [1], clause weighting dynamic local search (DLS) algorithms for SAT have been intensively investigated, and continually improved $[2,3]$. However, the performance of these algorithms remained inferior to their non-weighting counterparts (e.g. [4]), until the more recent development of weight smoothing heuristics [5-8]). Such algorithms now represent the state-of-the-art for stochastic local search (SLS) methods on SAT problems. Interestingly, the most successful DLS algorithms (i.e. DLM [5], SAPS [7] and PAWS [8]) have converged on the same underlying weighting strategy: increasing weights on false clauses in a local minimum, then periodically reducing weights according to a problem specific parameter setting. DLM mainly differs from PAWS by incorporating a plateau searching heuristic and PAWS mainly differs from SAPS by performing additive rather than multiplicative weight updates. 
However, a key weakness of these approaches is that their performance depends on problem specific parameter tuning. This issue was partly addressed in the development of a reactive version of SAPS (RSAPS [7]) which used a similar adaptive noise mechanism to that used in AdaptNovelty ${ }^{+}$[9]. Nevertheless, as the 2005 International SAT competition (SAT2005) has shown, DLS algorithms, including RSAPS, have not proved competitive with the best SLS techniques when they are constrained to use fixed parameter values. This is explained by the sensitivity of the control parameters and by the lack of a sufficiently effective adaptive mechanism to adjust these parameters to specific problem instances.

In 2005, a new approach to clause weighting was developed, known as Divide and Distribute Fixed Weight (DDFW) [10]. DDFW's approach is to redistribute weight from satisfied to unsatisfied clauses in each local minimum, unifying the increase and decrease phases of weight control into a single action. This means there is no requirement for a problem specific parameter to decide when weight is to be reduced. In addition, DDFW only alters weights on those clauses that are false in a local minimum and an equal number of satisfied clauses. This makes it more efficient than earlier weight smoothing algorithms that also performed smoothing at each local minimum, but did so by adjusting weight on all the clauses in the problem (e.g. SDF [11]). However, DDFW still has a parameter $\left(W_{\text {init }}\right)$ whose setting can effect performance by varying the amount of weight that is initially given to each clause. In the earlier empirical evaluation of DDFW this initial weight was fixed. However, the existence of such a parameter implies that DDFW could benefit from an adaptive mechanism to vary the amount of weight that is distributed according to the dynamic search conditions.

Also in 2005, it was shown that the performance of various SLS techniques can be significantly improved by the addition of a resolution-based preprocessing phase [12]. This work initially produced the winning algorithm in the SAT2005 satisfiable random problem category, $\mathrm{R}+$ AdaptNovelty $^{+}$. However, in the subsequent paper [12], the largest performance gains were obtained for clause weighting algorithms solving structured problem instances. Here R+AdaptNovelty ${ }^{+}$ was convincingly outperformed by a $\mathrm{R}+\mathrm{RSAPS}$ and a tuned version of $\mathrm{R}+\mathrm{PAWS}$ on a range of quasigroup existence problems and standard structured SAT benchmarks.

The question we address in the current paper is which SLS SAT algorithm should be preferred in situations where parameter tuning is impractical and we have no other information that could guide us in choosing a particular approach. As this is exactly the situation we would expect to find in many real world applications, we take the relevance and importance of this question to be self evident. While the initial work on DDFW [10] showed that a fixed parameter version was able to outperform AdaptNovelty ${ }^{+}$and RSAPS on a range of random and structured SAT benchmarks, the question still remains whether the performance of DDFW can be further improved by incorporating a similar adaptive mechanism to that used by AdaptNovelty ${ }^{+}$and RSAPS to control the $W_{\text {init }}$ parameter.

It also remains unanswered whether such an adaptive version of DDFW could derive enough benefit from resolution-based preprocessing to outperform the ex- 
isting resolution-based versions of $\mathrm{R}+\mathrm{AdaptNovelty}^{+}$or R+RSAPS. In addition, in SAT2005 a new SLS algorithm was introduced, G²WSAT [13], which went on to win the silver medal in the random category of the competition. This algorithm has subsequently been improved and it too has yet incorporate a resolution-based preprocessor.

As a result of these considerations, our specific aim in the remainder of the paper is to introduce an adaptive resolution-incorporating version of DDFW (called $\mathrm{R}+\mathrm{DDFW}^{+}$) and to compare it with the three other most promising general purpose SLS SAT solvers, namely $\mathrm{R}+$ AdaptNovelty $^{+}, \mathrm{R}+\mathrm{RSAPS}$ and an enhanced $R+G^{2}$ WSAT. On the basis of an empirical study that covers a range of problems from SAT2005, the quasigroup existence domain and the SATLIB benchmark library, we conclude that $\mathrm{R}+\mathrm{DDFW}^{+}$has the best overall performance of these methods, and that it derives significant benefits from its new adaptive mechanism.

\section{Clause Weighting for SAT}

Clause weighting local search algorithms for SAT follow the basic procedure of repeatedly flipping single literals that produce the greatest reduction in the sum of false clause weights. Typically, all literals are randomly initialized, and all clauses are given a fixed initial weight. The search then continues until no further cost reduction is possible, at which point the weight on all unsatisfied clauses is increased, and the search is resumed, punctuated with periodic weight reductions.

Existing clause weighting algorithms differ primarily in the schemes used to control the clause weights, and in the definition of the points where weight should be adjusted. Multiplicative methods, such as SAPS, generally adjust weights when no further improving moves are available in the local neighbourhood. This can be when all possible flips lead to a worse cost, or when no flip will improve cost, but some flips will lead to equal cost solutions. As multiplicative real-valued weights have much finer granularity, the presence of equal cost flips is much more unlikely than for an additive approach (such as DLM or PAWS), where weight is adjusted in integer units. This means that additive approaches frequently have the choice between adjusting weight when no improving move is available, or taking an equal cost (flat) move.

Despite these differences, the three most well-known clause weighting algorithms (DLM [5], SAPS [7] and PAWS [8]) share a similar structure in the way that weights are updated: ${ }^{4}$ Firstly, a point is reached where no further improvement in cost appears likely. The precise definition of this point depends on the algorithm, with DLM expending the greatest effort in searching plateau areas

\footnotetext{
${ }^{4}$ Additionally, a fourth clause weighting algorithm, GLSSAT [14], uses a similar weight update scheme, additively increasing weights on the least weighted unsatisfied clauses and multiplicatively reducing weights whenever the weight on any one clause exceeds a predefined threshold.
} 
of equal cost moves, and SAPS expending the least by only accepting cost improving moves. Then all three methods converge on increasing weights on the currently false clauses (DLM and PAWS by adding one to each clause and SAPS by multiplying the clause weight by a problem specific parameter $\alpha>1$ ). Each method continues this cycle of searching and increasing weight, until, after a certain number of weight increases, clause weights are reduced (DLM and PAWS by subtracting one from all clauses with weight $>1$ and SAPS by multiplying all clause weights by a problem specific parameter $\rho<1)$. SAPS is further distinguished by reducing weights probabilistically (according to a parameter $\left.P_{\text {smooth }}\right)$, whereas DLM and PAWS reduce weights after a fixed number of increases (again controlled by parameter). PAWS is mainly distinguished from DLM in being less likely to take equal cost or flat moves. DLM will take up to $\theta_{1}$ consecutive flat moves, unless all available flat moves have already been used in the last $\theta_{2}$ moves. PAWS does away with these parameters, taking flat moves with a fixed probability of $15 \%$, otherwise it will increase weight.

However, as we have stressed in the introduction, the performance of these clause weighting algorithms remains very sensitive to the settings of their problem specific parameters (this has been shown in detail in [15]). While this sensitivity is also a problem for the non-weighting algorithms of the WalkSAT family, it has been somewhat counteracted by the use of heuristics that adapt parameter settings during the course of the search. The most successful of these algorithms, AdaptNovelty ${ }^{+}$, works by adapting a noise parameter that controls whether a move is selected randomly or deterministically [9]. In simplified terms, the likelihood of making a random choice is increased the longer the search continues without achieving an improvement in the objective function. A similar scheme was added to SAPS, producing reactive SAPS or RSAPS [7]. However, adapting SAPS was not as successful as adapting Novelty, for, while a tuned SAPS generally produces better performance than a tuned Novelty+, RSAPS has not been able to reach the consistent performance AdaptNovelty ${ }^{+}$in the recent SAT competitions. One reason for this may be that SAPS requires the setting of three parameters to achieve its best performance, while RSAPS only adapts one of these parameters. Similarly, DLM requires the setting of at least three parameters before producing its best performance. In contrast, PAWS (like Novelty) only requires the tuning of a single parameter, but to date no successful heuristic has been discovered that can automatically adapt this value.

More recently, work has concentrated on learning empirical hardness models in order to predict the best parameter settings for SAPS [16]. This approach requires a set of training instances that are repeatedly solved by SAPS using different parameter settings. After this training phase, parameter settings can be generated for previously unseen instances taken from the same problem class. Results from this work are encouraging and could be generally applied to other local search algorithms. However, the weakness is that training is required on a representative test set before good predictions can be produced. It remains to be seen whether a general model can be devised that can predict good parameter settings for the SAT domain as a whole. In the meantime, if we are limited 
to solving problems from an undisclosed problem distribution and if manual parameter tuning is ruled out of court, then the best available clause weighting algorithm is probably RSAPS (discounting DDFW for the moment).

\section{Divide and Distribute Fixed Weights}

DDFW introduces two ideas into the area of clause weighting algorithms for SAT. Firstly, it evenly distributes a fixed quantity of weight across all clauses at the start of the search, and then escapes local minima by transferring weight from satisfied to unsatisfied clauses. The other existing state-of-the-art clause weighting algorithms have all divided the weighting process into two distinct steps: i) increasing weights on false clauses in local minima and ii) decreasing or normalising weights on all clauses after a series of increases, so that weight growth does not spiral out of control. DDFW combines this process into a single step of weight transfer, thereby dispensing with the need to decide when to reduce or normalise weight. In this respect, DDFW is similar to the predecessors of SAPS (SDF [6] and ESG [11]), which both adjust and normalise the weight distribution in each local minimum. Because these methods adjust weight across all clauses, they are considerably less efficient than SAPS, which normalises weight after visiting a series of local minima. ${ }^{5}$ DDFW escapes the inefficiencies of SDF and ESG by only transferring weights between pairs of clauses, rather than normalising weight on all clauses. This transfer involves selecting a single satisfied clause for each currently unsatisfied clause in a local minimum, reducing the weight on the satisfied clause by an integer amount and adding that amount to the weight on the unsatisfied clause. Hence DDFW retains the additive (integer) weighting approach of DLM and PAWS, and combines this with an efficient method of weight redistribution, i.e. one that keeps all weight reasonably normalised without repeatedly adjusting weights on all clauses.

DDFW's weight transfer approach also bears similarities to the operations research subgradient optimisation techniques discussed in [11]. In these approaches, Lagrangian multipliers, analogous to the clause weights used in SAT, are associated with problem constraints, and are adjusted in local minima so that multipliers on unsatisfied constraints are increased and multipliers on satisfied constraints are reduced. This symmetrical treatment of satisfied and unsatisfied constraints is mirrored in DDFW, but not in the other SAT clause weighting approaches (which increase weights and then adjust). However, DDFW differs from subgradient optimisation in that weight is only transferred between pairs of clauses and not across the board, meaning less computation is required.

\subsection{Exploiting Neighbourhood Structure}

The second and more original idea developed in DDFW, is the exploitation of neighbourhood relationships between clauses when deciding which pairs of clauses will exchange weight.

\footnotetext{
${ }^{5}$ Increasing weight on false clauses in a local minimum is efficient because only a small proportion of the total clauses will be false at any one time.
} 


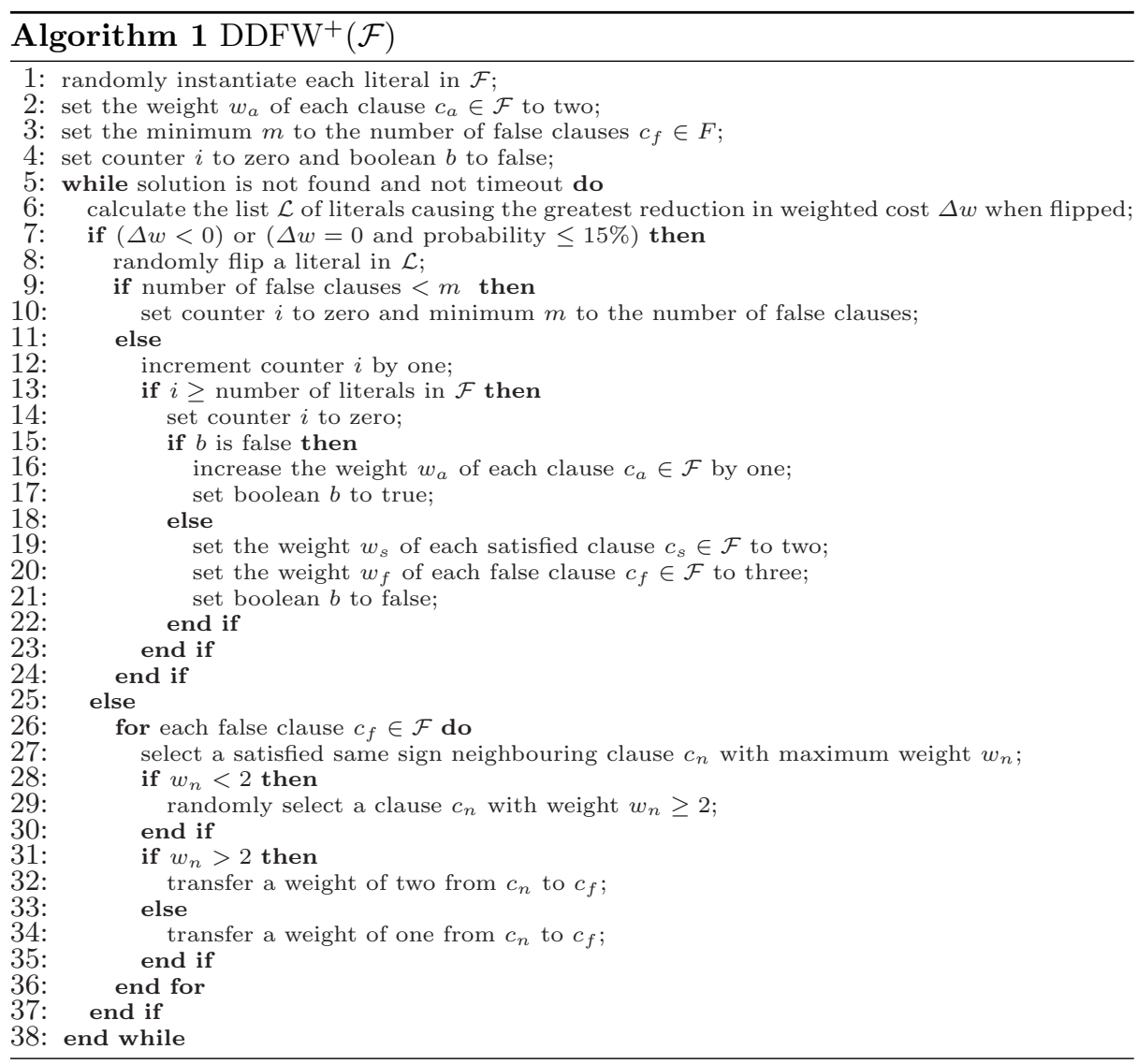

We term clause $c_{i}$ to be a neighbour of clause $c_{j}$, if there exists at least one literal $l_{i m} \in c_{i}$ and a second literal $l_{j n} \in c_{j}$ such that $l_{i m}=l_{j n}$. Furthermore, we term $c_{i}$ to be a same sign neighbour of $c_{j}$ if the sign of any $l_{i m} \in c_{i}$ is equal to the sign of any $l_{j n} \in c_{j}$ where $l_{i m}=l_{j n}$. From this it follows that each literal $l_{i m} \in c_{i}$ will have a set of same sign neighbouring clauses $C_{l_{i m}}$. Now, if $c_{i}$ is false, this implies all literals $l_{i m} \in c_{i}$ evaluate to false. Hence flipping any $l_{i m}$ will cause it to become true in $c_{i}$, and also to become true in all the same sign neighbouring clauses of $l_{i m}$, i.e. $C_{l_{i m}}$. Therefore, flipping $l_{i m}$ will help all the clauses in $C_{l_{i m}}$, i.e. it will increase the number of true literals, thereby increasing the overall level of satisfaction for those clauses. Conversely, $l_{i m}$ has a corresponding set of opposite sign clauses that would be damaged when $l_{i m}$ is flipped.

The reasoning behind the DDFW neighbourhood weighting heuristic proceeds as follows: if a clause $c_{i}$ is false in a local minimum, it needs extra weight in order to encourage the search to satisfy it. If we are to pick a neighbouring clause $c_{j}$ that will donate weight to $c_{i}$, we should pick the clause that is most able to pay. Hence, the clause should firstly already be satisfied. Secondly, it should be a same sign neighbour of $c_{i}$, as when $c_{i}$ is eventually satisfied by flipping 
$l_{i m}$, this will also raise the level of satisfaction of $l_{i m}$ 's same sign neighbours. However, taking weight from $c_{j}$ only increases the chance that $c_{j}$ will be helped when $c_{i}$ is satisfied, i.e. not all literals in $c_{i}$ are necessarily shared as same sign literals in $c_{j}$, and a non-shared literal may be subsequently flipped to satisfy $c_{i}$. The third criteria is that the donating clause should also have the largest store of weight within the set of satisfied same sign neighbours of $c_{i}$

The intuition behind the DDFW heuristic is that clauses that share same sign literals should form alliances, because a flip that benefits one of these clauses will always benefit some other member(s) of the group. Hence, clauses that are connected in this way will form groups that tend towards keeping each other satisfied. However, these groups are not closed, as each clause will have clauses within its own group that are connected by other literals to other groups. Weight is therefore able to move between groups as necessary, rather than being uniformly smoothed (as in existing methods).

\subsection{Adapting DDFW}

The new feature introduced in this study is the development of an adaptive mechanism that alters the total amount of weight that DDFW distributes according to the degree of stagnation in the search. This $\mathrm{DDFW}^{+}$heuristic is detailed in lines 9-24 of Algorithm 1. Previously DDFW would have initialised the weight of each clause to $W_{i n i t}$ (which was fixed at 8 in [10]). Now this initialisation value is set at two in line 2 of Algorithm 1, but can be altered during the search as follows: if the search executes a consecutive series of $i$ flips without reducing the total number of false clauses, where $i$ is equal to the number of literals in the problem, then the amount of weight on each clause is increased by one in the first instance. However, if after increasing weights, the search enters another consecutive series of $i$ flips without improvement, then it will reset the weight on each satisfied clause back to two and on each false clause back to three. The search then continues to follow each increase with a reset and each reset with an increase. In this way a long period of stagnation will produce oscillating phases of weight increase and reduction, such that the total weight can never exceed 3 times the total number of clauses $c_{a} \in \mathcal{F}$ plus the total number of false clauses $c_{f} \in \mathcal{F}$.

The reasoning behind this adaptive heuristic is based on our observation that manually adjusting DDFW's original parameter $W_{\text {init }}$ has a noticeable effect runtime performance, and that on several problems the default value of eight was not optimal. This is illustrated in Figure 1, which shows that on problem (a) $W_{\text {init }}=8$ is near optimal whereas on problem (b) $W_{\text {init }}=2$ is the better choice (if we consider the underlying trend). We conjectured that we could circumvent the need to initialise the clauses with more weight at the start of the search by allowing context sensitive weight increases during the search. Hence we developed a stagnation measure, much like the measures used in AdaptNovelty and RSAPS, that injects extra weight when no cost improvement occurs and made the frequency of this injection depend on the size of the problem. The unusual feature of the $\mathrm{DDFW}^{+}$heuristic is that the search will only effect one 
increase after which, if stagnation is observed again, the weights are reset. This reset mechanism was adopted after a series of empirical trials that tested various combinations of weight increase and decrease phases. Our main difficulty was to keep the weight growth within bounds and we could find no decrease scheme that worked well across a wide range of problems without requiring a further problem dependent parameter (which would obviously defeat the purpose of the study). We therefore settled on a simple reset strategy that places a strict limit on weight growth and avoids adding an additional parameter.
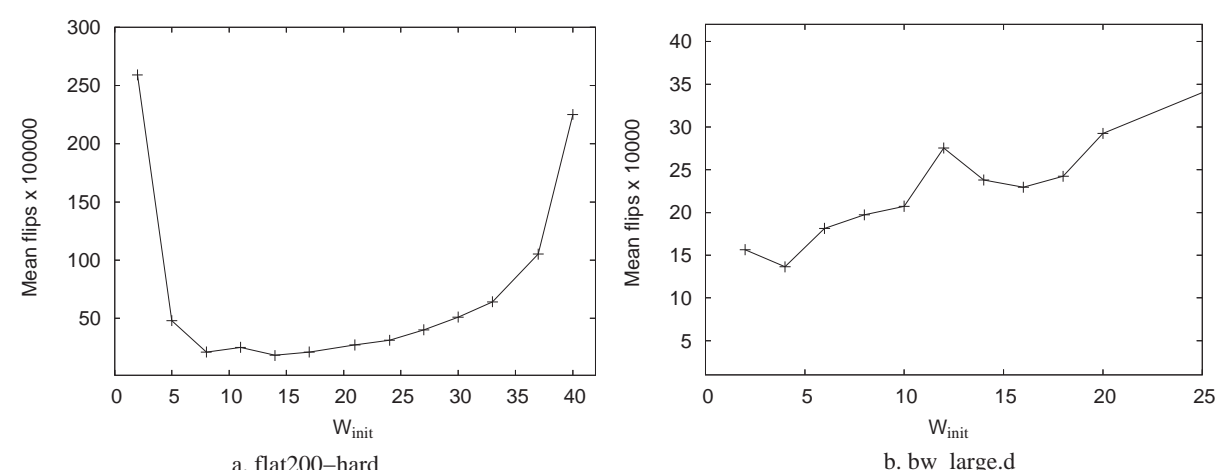

Fig. 1. Flip performance of DDFW for various settings of the $W_{\text {init }}$ parameter

\section{Resolution Based Preprocessing}

As discussed in the introduction, significant performance benefits have been gained by preprocessing a problem using resolution before starting a search. This result is already well-known in the complete search community, where Satz [17] uses a restricted resolution procedure, adding resolvents of length $\leq 3$, as a preprocessor before running the complete backtrack search. The same procedure has now been added to AdaptNovelty ${ }^{+}$, PAWS, RSAPS and WalkSAT [12], and there is empirical evidence to suggest that clause weighting algorithms in particular benefit from this approach when solving structured real-world problems.

Resolution itself is a rule of inference widely used in automated deduction [1820]. In the present study, as in [12], we implement the Satz resolution process (see Algorithm 2) as follows: when two clauses of a CNF formula have the property that some variable $x_{i}$ occurs positively in one and negatively in the other, the resolvent of the clauses is a disjunction of all the literals occurring in the clauses except $x_{i}$ and $\overline{x_{i}}$. For example, the clause $\left(x_{2} \vee x_{3} \vee \overline{x_{4}}\right)$ is the resolvent for the clauses $\left(\overline{x_{1}} \vee x_{2} \vee x_{3}\right)$ and $\left(x_{1} \vee x_{2} \vee \overline{x_{4}}\right)$ and is added to the clause set. The new clauses, provided they are of length $\leq 3$, can in turn be used to produce other resolvents. The process is repeated until saturation. Duplicate and subsumed 


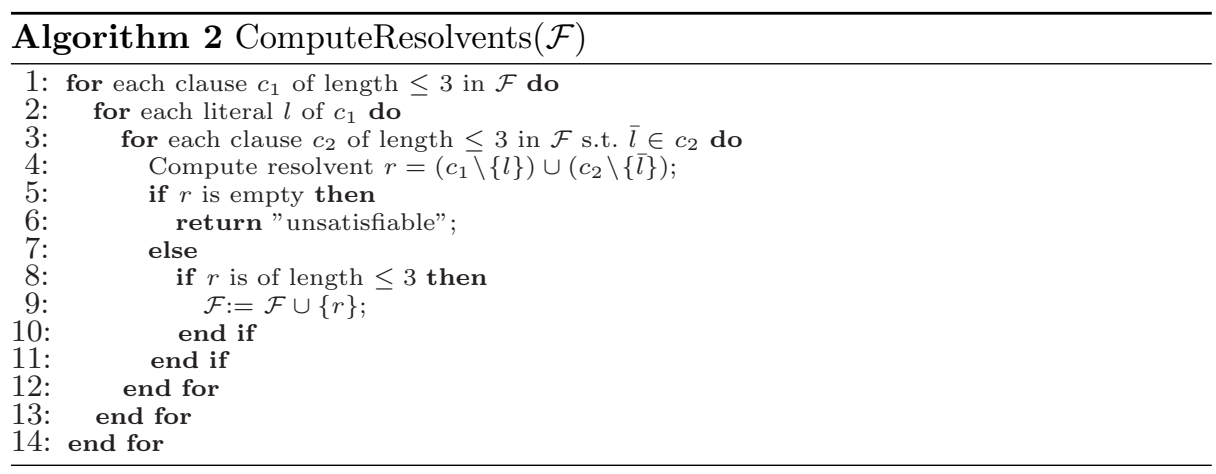

clauses are deleted, as are tautologies and any duplicate literals in a clause. It is worth noting that this resolution phase takes polynomial time.

\section{Experimental Evaluation}

As the resolution process is encapsulated in a preprocessing phase, it can be added to an existing SAT solver as a separate module, leaving the original solver unaltered. In our experimental study we added this preprocessing phase (as defined in Algorithm 2) to DDFW, DDFW ${ }^{+}$, RSAPS, AdaptNovelty ${ }^{+}$and $\mathrm{G}^{2} \mathrm{WSAT}$, producing $\mathrm{R}+\mathrm{DDFW}, \mathrm{R}+\mathrm{DDFW}^{+}, \mathrm{R}+\mathrm{RSAPS}, \mathrm{R}+$ AdaptNovelty ${ }^{+}$ and $\mathrm{R}+\mathrm{G}^{2}$-WSAT. Of these algorithms, $\mathrm{R}+\mathrm{RSAPS}$ and $\mathrm{R}+$ AdaptNovelty ${ }^{+}$have already been entered into SAT2005 and reported in [12]. ${ }^{6}$ However, R+DDFW, $\mathrm{R}+\mathrm{DDFW}^{+}$and $\mathrm{R}+\mathrm{G}^{2} \mathrm{WSAT}$ are new algorithms whose performance has yet to be reported. ${ }^{7}$ We chose to compare DDFW with $\mathrm{R}+$ AdaptNovelty ${ }^{+}$and $\mathrm{R}+\mathrm{G}^{2}$ WSAT because these two algorithms were the gold and silver medal winners in the SAT2005 satisfiable random category competition and achieved the best overall local search results in terms of the number of problems solved. We chose R+RSAPS because it was the best performing clause weighting algorithm in the competition. Together, therefore, these three algorithms can lay claim to being the state-of-the-art for general purpose local search SAT solving when manual parameter tuning is disallowed.

To evaluate the relative performance of these algorithms we divided our empirical study into four areas: firstly, we attempted to reproduce a reduced problem set similar to that used in the random category of the SAT competition (as this is the domain where local search techniques have dominated). To do this we selected the 50 satisfiable $k 3$ problems from the SAT2004 competition

\footnotetext{
${ }^{6}$ AdaptNovelty ${ }^{+}$and RSAPS are available as part of the UBCSAT solver from http://www.satlib.org/ubcsat/

${ }^{7} \mathrm{G}^{2}$ WSAT is available at http://www.laria.u-picardie.fr/\%7Ecli/g2wsat2005.c. This latest version is described by the authors as generally more than $50 \%$ faster than the version entered in SAT2005.
} 
benchmark. Secondly, we obtained the 10 SATLIB quasigroup existence problems used in [12]. These problems are relevant because they exhibit a balance between randomness and structure, while also producing clause sets to which resolution can be applied effectively. Thirdly, we obtained the structured problem set used to originally evaluate SAPS [7]. These problems have been widely used to evaluate clause weighting algorithms (e.g. in [8]) and contain a representative cross-section taken from the DIMACS and SATLIB libraries. In this set we also included 4 of the well-known DIMACS 16-bit parity learning problems. Finally, we used the 16 ferry planning problems from the SAT2005 competition that our local search techniques were able to solve. This was to give an indication of relative performance on the SAT2005 industrial problems.

Overall, the problem set is designed to show how $\mathrm{R}+\mathrm{DDFW}^{+}$compares in absolute terms to the other algorithms and to examine the relative effect of the adaptive mechanism on differing problem classes. For this reason we also include the results for $\mathrm{R}+\mathrm{DDFW}$ (i.e. without the adaptive mechanism). All experiments were performed on a Dell machine with $3.1 \mathrm{GHz}$ CPU and 1GB memory, except for the quasigroup problems which were run on a Sun supercomputer with $8 \times$ Sun Fire V880 servers, each with $8 \times$ UltraSPARC-III 900MHz CPU and 8 GB memory per node. Cut-offs for the various algorithms were set as follows: first $\mathrm{R}+\mathrm{DDFW}$ was given 10 trials on each problem with a flip cut-off of $1,000,000$. If it was unable to solve any trial then the cut-off was raised to $10,000,000$, and then in steps of 10,000,000 until at least one solution was found. R+DDFW was then allowed 100 trials at the given flip cut-off for all instances except the ferry problems, where it was limited to 10 trials. The total time allowed for R+DDFW on each set of 10 or 100 trials was then recorded and all other algorithms were given this as a time cut-off on each problem. The following results detail the mean time in seconds (including the resolution preprocessing step), mean flips and the success rate for these cut-offs (results in bold indicate the best performance for a particular problem).

\subsection{SAT Competition Problem Results}

The results in Figure 2a graph the performance of $\mathrm{R}+\mathrm{DDFW}^{+}, \mathrm{R}+\mathrm{DDFW}$, $\mathrm{R}+$ AdaptNovelty $^{+}$and $\mathrm{R}+\mathrm{G}^{2} \mathrm{WSAT}$ after applying resolution on the $50 \mathrm{k3}$ problems from the SAT2004 competition (as R+RSAPS had very poor performance on the random instances it has been omitted from the figure and the following discussion). The graph shows the cumulative percentage of problems solved against runtime, assuming that each instance is solved in parallel (for example, in Figure 2a after 5 seconds approximately $71 \%$ of the $50 \times 100$ trials for R+DDFW will have terminated). Here $\mathrm{R}+\mathrm{DDFW}^{+}$and $\mathrm{R}+\mathrm{DDFW}$ were the only solvers that could reach a $100 \%$ success rate over all trials. Although $\mathrm{R}+\mathrm{G}^{2} \mathrm{WSAT}$ was competitive and could solve the easier problems faster than $\mathrm{R}+\mathrm{DDFW}$, it was unable to match $\mathrm{R}+\mathrm{DDFW}$ as problem difficulty increased. Overall the graph shows that $\mathrm{R}+\mathrm{DDFW}^{+}$has the superior performance across the range of problem sizes, clearly dominating $\mathrm{R}+\mathrm{DDFW}$ and 

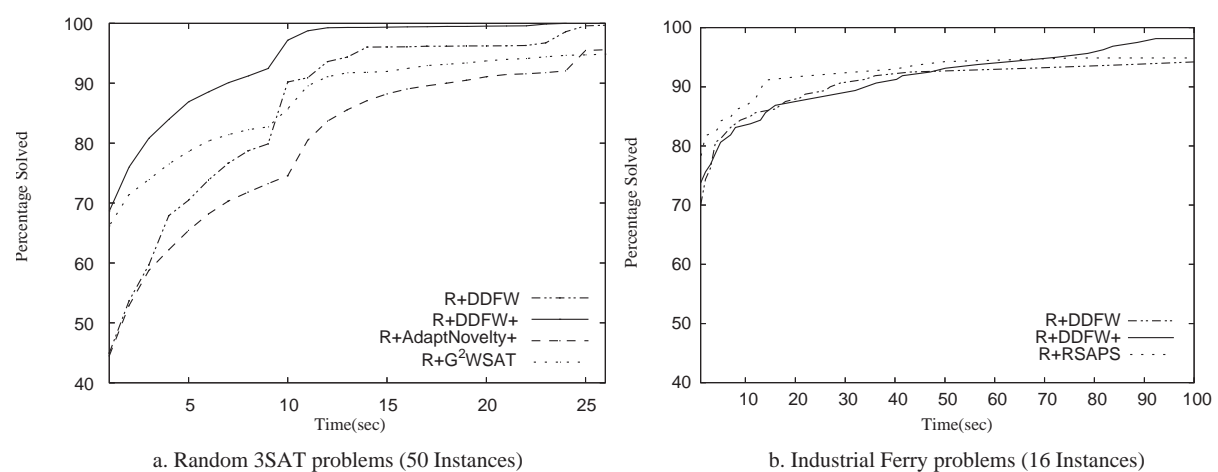

Fig. 2. Results for the SAT2004 random problems and SAT2005 industrial problems

thereby demonstrating that the new adaptive heuristic can positively affect runtime performance. Figure $2 \mathrm{a}$ also shows that $\mathrm{R}+\mathrm{G}^{2} \mathrm{WSAT}$ generally dominates $\mathrm{R}+$ AdaptNovelty $^{+}$, although $\mathrm{R}+$ AdaptNovelty ${ }^{+}$does match $\mathrm{R}+\mathrm{G}^{2} \mathrm{WSAT}$ 's success rate over the whole problem set.

The results for the SAT2005 industrial ferry problems are shown in Figure $2 \mathrm{~b}$ and in Table 1 (as $\mathrm{R}+\mathrm{G}^{2} \mathrm{WSAT}$ and $\mathrm{R}+$ AdaptNovelty ${ }^{+}$were only able to solve $29 \%$ and $9 \%$ of the ferry instances respectively, they have been removed from the graphical analysis). Looking at Figure $2 \mathrm{~b}$ we can see that $\mathrm{R}+\mathrm{RSAPS}$, after performing poorly on the random problems, is now able to dominate $\mathrm{R}+\mathrm{DDFW}$ across the range of the ferry problems, but cannot quite reach $\mathrm{R}+\mathrm{DDFW}{ }^{+}$'s $97.5 \%$ success rate. However, Table 1 shows that $\mathrm{R}+\mathrm{RSAPS}$ is able to solve 10 of the 16 ferry problems faster than either DDFW variant, and that $\mathrm{R}+\mathrm{DDFW}^{+}$'s superior success rate is largely based on instance ferry 4001 . We must therefore conclude that there is little to choose between $\mathrm{R}+\mathrm{RSAPS}$ and $\mathrm{R}+\mathrm{DDFW}^{+}$on these problems. Nevertheless, $\mathrm{R}+\mathrm{DDFW}^{+}$does more clearly outperform $\mathrm{R}+\mathrm{DDFW}$ and again demonstrates that the adaptive heuristic can make noticeable improvements.

\subsection{Quasigroup Problem Results}

Table 2 shows the performance of the solvers on the quasigroup problems. Here we can see that $\mathrm{R}+\mathrm{DDFW}$ and $\mathrm{R}+\mathrm{DDFW}^{+}$clearly emerge as the two best solvers, sharing the best results for each instance and both achieving an overall success rate of $100 \%$. Comparing between the two DDFW methods, for the first time it becomes unclear whether the adaptive heuristic has made any difference, as, for most instances the results are comparable. However $\mathrm{R}+\mathrm{DDFW}^{+}$does exhibit noticeably better performance on instance qg1-08, whereas $\mathrm{R}+\mathrm{DDFW}$ shows equally strong performance on qg7-13. We should therefore conclude that the adaptive mechanism does not change the overall performance of DDFW on this problem set, although it can make a difference, either positively or negatively, on individual instances. 


\begin{tabular}{|c|c|c|c|c|c|c|c|c|c|c|c|c|c|c|c|}
\hline & \multicolumn{3}{|c|}{${\mathrm{R}+\mathrm{DDFW}^{+}}^{+}$} & \multicolumn{3}{|c|}{$\mathrm{R}+\mathrm{DDFW}$} & \multicolumn{3}{|c|}{$\mathrm{R}+$ AdaptNovelty ${ }^{+}$} & \multicolumn{3}{|c|}{$\mathrm{G}^{2}$ WSAT } & \multicolumn{3}{|c|}{ R+RSAPS } \\
\hline is & Time & Flips & $\%$ & $\mathrm{me}$ & Flips & $\%$ & Time & Flips & $\%$ & Time & Flips & \begin{tabular}{|l|}
$\%$ \\
\end{tabular} & Time & Flips & \\
\hline rry & 3.48 & 73,195 & 100 & .1 & 6,967 & 100 & $n / a$ & $n / a$ & & $n / a$ & $n / a$ & ct & 5.6 & 0,501 & \\
\hline & & & 1 & & 8,302 & 100 & $n / a$ & $n / a$ & & $n / c$ & $n / a$ & & .1 & 30 & \\
\hline 19 & & 903 & 100 & 0. & 3,942 & 100 & 3.9 & 511 & 20 & 1 & 5,5471 & 100 & .0 &, 741 & 10 \\
\hline ry & & 38,690 & 60 & .3 & 55,539 & 90 & $n / a$ & $n / a$ & & $/ a$ & $n / a$ & 0 & 9.2 & 42,006 & \\
\hline erry & & 526 & 100 & 0 & 8,586 & 100 & 2.13 & 936 & 00 & 0.1 & \begin{tabular}{l|l|l|}
, 334 & 1 \\
\end{tabular} & 100 & .0 & 5,070 & 010 \\
\hline erry & 1 & 70 & 1 & 3.2 & 08,547 & 100 & $n / a$ & $n / c$ & 0 & $n / a$ & $n / a$ & 0 & .6 & 04,680 & 10 \\
\hline$r$ & 0 & 774 & 100 & 0.0 & 19,280 & 100 & $|a|$ & $n$ & 0 & 1.8 & $2,442,300$ & 80 & 0.0 & 2,771 & 10 \\
\hline 01 & 63.1 & $, 392,288$ & 100 & 99.4 & $40,117,368$ & 90 & $n / a$ & $n / a$ & 0 & $n / a$ & $n / a$ & 0 & 90.05 & $54,061,467$ & \\
\hline ferry & 0.0 & 9,637 & 100 & 0.0 & 20,336 & 100 & 4.87 & 284 & 30 & 2.1 & $1,958,552$ & 90 & 0.0 & 3,852 & 210 \\
\hline $\mathrm{rry}_{3}$ & 21.2 & 395,968 & 100 & 21.2 & 773,439 & 50 & $n / a$ & $n /$ &  & $n / a$ & $n / a$ & 0 & 7.2 & $2,884,301$ & 110 \\
\hline erry & 0 & 348 & 100 & 1 &, 547 & 100 & $n / a \mid$ & $n_{1}$ & 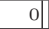 & 2.4 & $2,437,826$ & 50 & .0 & 20,394 & \begin{tabular}{l|l}
4 & 10 \\
\end{tabular} \\
\hline 006 & 0.0 & 640 & 100 & 0] & 7,697 & 100 & $n / a$ & $n /$ & U & 4.9 & $2,616,491$ & 20 & 0.0 & 9,160 & 10 \\
\hline ry 4008 & 0 & 192 & 100 & 0.1 & ,796 & 100 & $n / a$ & $n /$ & 0 & 3.2 & $2,655,066$ & 20 & 1.1 & 2,938 & 810 \\
\hline $\mathrm{ry}$ & $\overline{0}$ & 163 & 100 & 0.1 &, 015 & 100 & $n / a$ & $n / a$ & 0 & $n / a$ & $n / a$ & 0 & 1 & 7,612 & 210 \\
\hline 92 & 1 & 525 & 100 & 0.2 & 102,413 & 100 & $t$ & & & 2/a & $n / a$ & 0 & .2 & 2,346 & \begin{tabular}{|l|l|}
6 & 10 \\
\end{tabular} \\
\hline rry3993 & 0.0 & 8,878 & 100 & 0.1 & 43,595 & 100 & $n / a$ & $n / a$ & 0 & 7.2 & $3,399,169$ & 10 & 0.2 & 54,742 & \begin{tabular}{|l|l|}
2 & 10 \\
\end{tabular} \\
\hline
\end{tabular}

Table 1. Results for the SAT2005 industrial ferry planning problems

\begin{tabular}{|c|c|c|c|c|c|c|c|c|c|c|c|c|c|c|c|}
\hline & \multicolumn{3}{|c|}{${\mathrm{R}+\mathrm{DDFW}^{+}}^{+}$} & \multicolumn{3}{|c|}{$\mathrm{R}+\mathrm{DDFW}$} & \multicolumn{3}{|c|}{ R+AdaptNovelty ${ }^{+}$} & \multicolumn{3}{|c|}{$\mathrm{R}+\mathrm{G}^{2} \mathrm{WSAT}$} & \multicolumn{3}{|c|}{ R+RSAPS } \\
\hline oblems & Time & Flips & $\%$ & Time & Flips & $\%$ & Time & Flips & $\%$ & Time & Flips & $\%$ & Time & Flips & $\%$ \\
\hline qg1-07 & 0.0 & 4,388 & 100 & 0.1 & 11,375 & 100 & 0.2 & 14,840 & 100 & 0.1 & 9,600 & 100 & 0.1 & 4,9011 & 100 \\
\hline qg1-0 & 10.2 & 2,276 & 100 & 21.8 & 601,271 & 100 & 33.8 & $1,076,689$ & 100 & 28.8 & $2,818,904$ & 100 & 64.6 & $2,153,008$ & 99 \\
\hline qg2-0 & 0.0 & 2,361 & 100 & 0.0 & $\begin{array}{ll}2,035 & 1 \\
\end{array}$ & 100 & 0.1 & 9,094 & 100 & 0.1 & 5,073 & 100 & 0.1 & $2,478 \mid 1$ & 100 \\
\hline qg2-08 & 57.5 & $1,556,545$ & 100 & 60.01 & $1,346,438$ & 100 & 77.1 & $1,906,196$ & 20 & 79.8 & $4,569,088$ & 50 & 71.5 & $1,879,019$ & 70 \\
\hline qg 3 & 0.1 & 16,867 & 100 & 0.1 & 21,986 & 100 & 0.6 & 78,849 & 100 & 0.1 & 24,534 & 100 & 0.2 & 1,0491 & 100 \\
\hline $\mathrm{qg} 4-0 \mathrm{~s}$ & 0.2 & 311 & 100 & 0.2 & 26,123 & 100 & 1.5 &, 169 & 100 & 0.7 & 142,619 & 100 & 1.2 & 4,9201 & 100 \\
\hline qg5-1 & 0.2 & 7,303 & 100 & .2 & 6,797 & 100 & 2.3 & 1,924 & 100 & 0.4 &, 992 & 100 & 0.6 & $\begin{array}{l}1,014 \\
1,1\end{array}$ & 100 \\
\hline qg6-09 & 0.0 & 478 & 100 & 0.0 & 4661 & 100 & 0.0 & 3,644 & 100 & 0.0 & 686 & 100 & 0.6 & 1,7531 & 100 \\
\hline qg7-09 & 0.0 & 292 & 100 & 0.0 & 299 & 100 & 0.0 & 698 & 100 & 0.0 & 412 & 100 & 0.0 & 295 & 100 \\
\hline qg7-13 & 9.3 & 229,258 & 100 & 3.2 & 122,091 & 100 & 16.3 & $5,351,459$ & 56 & $n / a$ & $n / a$ & 0 & 24.9 & 373,456 & 10 \\
\hline
\end{tabular}

Table 2. Results for Quasigroup SATLIB problems

\subsection{Structured Problem Results}

Table 3 shows the results for the structured problems taken from the original SAPS problem set [7] and the parity learning problems taken from the original PAWS study [8]. This set comprises of two blocks world planning (bw) problems, two logistics planning instances, two flat graph coloring problems (flat), two all-interval-series problems (ais) and four 16-bit parity learning problems (par16*). The results confirm our earlier observation from the random problem results that $\mathrm{G}^{2} \mathrm{WSAT}$ does not scale as well as DDFW. In this case $\mathrm{R}+\mathrm{G}^{2} \mathrm{WSAT}$ is the best algorithm on the smaller ais, logistics and flat problems, but is outperformed by $\mathrm{R}+\mathrm{DDFW}$ on each of the larger instances of these problems. In addition, $\mathrm{R}+\mathrm{RSAPS}$ has stronger performance than $\mathrm{R}+\mathrm{DDFW}$ on the ais and par16 problems.

However, the situation changes if we consider the performance of $\mathrm{R}+\mathrm{DDFW}^{+}$. In comparison to $\mathrm{R}+\mathrm{DDFW}, \mathrm{R}+\mathrm{DDFW}^{+}$is better on the ais10, both logistics and all par16 problems, whereas R+DDFW is only better on the ais12 and flat200 problems (the two methods perform identically on the bw problems be- 
cause the large number of literals mean the adaptive mechanism is not used). These results show that the $\mathrm{R}+\mathrm{DDFW}^{+}$adaptive mechanism has again produced noticeable performance benefits, and has improved the overall behaviour of $\mathrm{R}+\mathrm{DDFW}$ on this problem set. In addition, if we take a simple count of the number of problems on which $\mathrm{R}+\mathrm{DDFW}^{+}$dominates we can see that it is also the best of the five algorithms considered.

\begin{tabular}{|c|c|c|c|c|c|c|c|c|c|c|c|c|c|c|}
\hline & \multicolumn{2}{|c|}{$\mathrm{R} \mathrm{DDFW}^{+}$} & \multicolumn{3}{|c|}{$\mathrm{R}+\mathrm{DDFW}$} & \multicolumn{3}{|c|}{$\mathrm{R}+$ Adapt Novelty $+\mid$} & \multicolumn{3}{|c|}{$\mathrm{R}+\mathrm{G}^{2} \mathrm{WSAT}$} & \multicolumn{3}{|c|}{$\mathrm{R}+\mathrm{RSAPS}$} \\
\hline Problems & Time & \begin{tabular}{l|l|} 
Flips & $\%$ \\
\end{tabular} & Time & Flips & $\%$ & Time & \begin{tabular}{l|l|} 
Flips \\
\end{tabular} & $\%$ & Time & \begin{tabular}{l|l|} 
Flips \\
\end{tabular} & $\%$ & Time & Flips & $\%$ \\
\hline ais 10 & 0.0 & \begin{tabular}{|l|l|}
298,650 & 100 \\
\end{tabular} & 0.5 & 498,911 & 100 & 1.4 & $1,214,321$ & 100 & 0.0 & 112,0441 & 100 & 0.0 & 25,459 & \\
\hline ais12 & 5.0 & \begin{tabular}{|l|l|}
$4,036,866$ & 100 \\
\end{tabular} & 2.3 & $1,934,170$ & 100 & 10.1 & 328,426 & 51 & 2.4 & $1,854,652$ & 100 & 0.2 & 37,743 & \\
\hline logistic: & 0.0 & \begin{tabular}{|l|l|}
242,540 & 100 \\
\end{tabular} & 0.3 & 4,645 & 100 & 0.0 & 26,696 & 100 & 0.0 & 23,6231 & 100 & 0.0 & 5,364 & \\
\hline logistics- & 0.1 & 16,708100 & 0.1 &, 86 & 100 & 0.1 & 99,650 & 100 & 0.5 & 350,711 & 100 & 0.1 & 20,918 & \\
\hline flat200-1 & 0.3 & \begin{tabular}{|l|l|}
262,905 & 100 \\
\end{tabular} & 0.2 & 161,902 & 100 & 0.2 & 51,563 & 100 & 0.1 & 150,5881 & 100 & 0.4 & 362,786 & 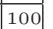 \\
\hline flat200-h & 3.2 & \begin{tabular}{|l|l|}
$2,814,221$ & 100
\end{tabular} & $1.0 \mid$ & 014,878 & 100 & 3.6 & , 166,964 & 36 & 2.4 & 535,185 & 100 & 3.5 & $, 517,562$ & \\
\hline bw_large.c & $=$ & $=100$ & 0.6 & 145,607 & 100 & 6.7 & $5,660,460$ & 67 & $n / a$ & $n / a$ & 0 & 21.3 & $4,258,483$ & \\
\hline bw_large.d & $=$ & $=100$ & 1.4 & 184,874 & 100 & 13.4 & $7,974,818$ & 38 & $n / a$ & $n / a$ & 0 & $n / a$ & $n / a$ & \\
\hline par16-1 & \begin{tabular}{l|l}
4.3 & 3 \\
\end{tabular} & \begin{tabular}{|l|l|}
$, 828,086$ & 100 \\
\end{tabular} & 7.1 & $5,229,852$ & 50 & \begin{tabular}{ll|l}
7.41 \\
\end{tabular} & $15,608,349$ & 15 & $n / a$ & $n / a$ & 0 & 7.4 & $1,164,862$ & \\
\hline par16-2 & $\begin{array}{ll}23.22 \\
\end{array}$ & \begin{tabular}{|l|l|l|l|l|}
$1,670,517$ & 100 \\
\end{tabular} & 27.92 & $20,542,514$ & 60 & 36.85 & $54,634,563$ & 10 & $n / a$ & $n / a$ & 0 & 16.01 & $7,581,843$ & 10 \\
\hline par16-3 & 7.77 & \begin{tabular}{ll|}
$, 146,517100$ \\
\end{tabular} & 24.41 & $17,959,087$ & \begin{tabular}{l||}
70 \\
\end{tabular} & 32.75 & $50,828,991$ & 40 & 31.8 & $26,133,070$ & 30 & 16.0 & $18,890,265$ & 10 \\
\hline par16-4 & \begin{tabular}{l|l}
2.9 & 2 \\
\end{tabular} & \begin{tabular}{|l|l|l|}
$2,699,444$ & 100 \\
\end{tabular} & 11.41 & $12,800,152$ & 100 & 26.84 & $41,099,634$ & 50 & 26.5 & $51,205,540$ & 60 & 8.1 & $9,445,556$ & 10 \\
\hline
\end{tabular}

Table 3. Results for structured problems from the SAPS and PAWS original studies, (the $=$ symbol means that $\mathrm{R}+\mathrm{DDFW}^{+}$behaves identically to $\mathrm{R}+\mathrm{DDFW}$ on these problems)

\section{Analysis and Conclusions}

Overall we can conclude that the addition of an adaptive mechanism has improved the performance of DDFW over the entire range of the problem sets we have considered. The strongest dominance was observed on the random 3-SAT and parity problems (shown in Figure $2 \mathrm{a}$ and Table 3 respectively). On the other problems $\mathrm{R}+\mathrm{DDFW}^{+}$improved over $\mathrm{R}+\mathrm{DDFW}$ on 10 of the 16 ferry problems (in Table 1), 6 of the 10 quasigroup problems (in Table 2) and stays neutral on the remaining real-world problems (in Table 3 ).

We can further conclude that $\mathrm{R}+\mathrm{DDFW}$ (i.e. even without the adaptive mechanism) has the better overall performance in comparison to AdaptNovelty ${ }^{+}$, $\mathrm{G}^{2} \mathrm{WSAT}$ and $\mathrm{R}+\mathrm{RSAPS}$. If we first look at $\mathrm{R}+\mathrm{G}^{2} \mathrm{WSAT}$, while it performed well on the smaller random problems, it could not match $\mathrm{R}+\mathrm{DDFW}$ on the larger more difficult random problems. In the other categories $\mathrm{R}+\mathrm{G}^{2} \mathrm{WSAT}$ was less competitive, again showing promise on the smaller structured problems in Table 3, but failing to scale up as well as R+DDFW on the more difficult problems. Interestingly, $\mathrm{G}^{2}$ WSAT performed strongly on the quasigroup problems when no resolution was performed, but was uncompetitive after resolution (these results are not reported in the current paper). This confirms the findings in [12] that suggest clause weighting algorithms can gain more advantage from resolution 
than non-weighting algorithms. In addition, $\mathrm{R}+\mathrm{G}^{2} \mathrm{WSAT}$ was uniformly worse than $\mathrm{R}+\mathrm{DDFW}$ on the ferry problems.

Turning our attention to R+RSAPS, this algorithm showed slightly better performance than $\mathrm{R}+\mathrm{DDFW}$ on the structured and ferry problems, dominating on 10 of the 16 ferry problems and on all the parity problems, with $\mathrm{R}+\mathrm{DDFW}$ showing the better performance on the remaining 6 ferry problems and on the other larger structured problems. However, R+RSAPS was outperformed by $\mathrm{R}+\mathrm{DDFW}^{+}$on the parity problems, was uniformly worse on the random problems and was uncompetitive with $\mathrm{R}+\mathrm{DDFW}$ on the quasigroup problems, thereby failing to show the same robust performance as $\mathrm{R}+\mathrm{DDFW}$ and $\mathrm{R}+\mathrm{DDFW}^{+}$across the whole range of problem sets. Our third comparison algorithm, $\mathrm{R}+$ AdaptNovelty $^{+}$, also had the worst overall performance, being unable to achieve outright dominance on any of the problems considered.

In a further unpublished study (not reported here) we investigated the effect of the preprocessing resolution step on the performance of each algorithm. This showed that resolution has little effect on the random problem instances but has a positive effect on the quasigroup instances, with the effect being more pronounced for $\mathrm{R}+\mathrm{DDFW}$ and less pronounced for $\mathrm{R}+\mathrm{G}^{2} \mathrm{WSAT}$. For the real world instances, resolution was also generally helpful for the ferry, ais, logistics and parity problems but had little or no effect on the bw and flat problems.

In conclusion, we have introduced and integrated a new adaptive mechanism into the DDFW algorithm. This mechanism is unusual in that it oscillates between increasing and resetting clause weights, timing these changes according to a stagnation measure defined by the number of problem literals. While the increase mechanism increments the existing weight profile, the reset mechanism eliminates the profile entirely, returning the weights to their initial state. We conjecture that this dramatic and discontinuous change in the weighted cost surface increases diversity by allowing the search to explore new trajectories. The reset mechanism also ensures that the amount of weight added to a problem is strictly controlled without requiring an additional weight decrease parameter.

In order to evaluate the new adaptive algorithm, $\mathrm{R}+\mathrm{DDFW}^{+}$, we also incorporated the latest resolution-based preprocessing technique used by the winning algorithm in the SAT2005 competition. In a broad ranging empirical study we have shown that integrating our new adaptive mechanism into DDFW can significantly enhance its overall performance. We have also shown that $\mathrm{R}+\mathrm{DDFW}^{+}$ has the best overall performance across a range of representative structured and random problem instances in comparison to three of the best SLS solvers currently available. The results suggest that $\mathrm{R}+\mathrm{DDFW}^{+}$should be the SLS algorithm of choice in situations where the characteristics of a problem domain are not known in advance and manual parameter tuning is not practical. In future work it would be worthwhile to experiment with other resolution techniques to see if further performance benefits can be obtained.

Acknowledgement: The authors would like to acknowledge the financial support of National ICT Australia (NICTA) and the Queensland government. NICTA 
is funded through the Australian Government's Backing Australia's Ability initiative and also through the Australian Research Council.

\section{References}

1. Morris, P.: The Breakout method for escaping from local minima. In: Proceedings of 11th AAAI. (1993) 40-45

2. Cha, B., Iwama, K.: Adding new clauses for faster local search. In: Proceedings of 13th AAAI. (1996) 332-337

3. Frank, J.: Learning short-term clause weights for GSAT. In: Proceedings of 15th IJCAI. (1997) 384-389

4. McAllester, D., Selman, B., Kautz, H.: Evidence for invariants in local search. In: Proceedings of 14th AAAI. (1997) 321-326

5. Wu, Z., Wah, B.: An efficient global-search strategy in discrete Lagrangian methods for solving hard satisfiability problems. In: Proceedings of 17th AAAI. (2000) 310 315

6. Schuurmans, D., Southey, F.: Local search characteristics of incomplete SAT procedures. In: Proceedings of 10th AAAI. (2000) 297-302

7. Hutter, F., Tompkins, D., Hoos, H.: Scaling and Probabilistic Smoothing: Efficient dynamic local search for SAT. In: Proceedings of 8th CP. (2002) 233-248

8. Thornton, J., Pham, D.N., Bain, S., Ferreira Jr., V.: Additive versus multiplicative clause weighting for SAT. In: Proceedings of 19th AAAI. (2004) 191-196

9. Hoos, H.: An adaptive noise mechanism for WalkSAT. In: Proceedings of 19th AAAI. (2002) 655-660

10. Ishtaiwi, A., Thornton, J., Sattar, A., Pham, D.N.: Neighbourhood clause weight redistribution in local search for SAT. In: Proceedings of 11th CP. (2005) $772-$ 776

11. Schuurmans, D., Southey, F., Holte, R.: The exponentiated subgradient algorithm for heuristic boolean programming. In: Proceedings of 17th IJCAI. (2001) 334-341

12. Anbulagan, Pham, D., Slaney, J., Sattar, A.: Old resolution meets modern SLS. In: Proceedings of 20th AAAI. (2005) 354-359

13. Li, C.M., Huang, W.: Diversification and determinism in local search for satisfiability. In: Proceedings of 8th SAT. (2005) 158-172

14. Mills, P., Tsang, E.: Guided local search applied to the satisfiability (SAT) problem. In: Proceedings of 15th ASOR. (1999) 872-883

15. Thornton, J.: Clause weighting local search for SAT. Journal of Automated Reasoning (2006) (to appear)

16. Hutter, F., Hamadi, Y.: Parameter adjustment based on performance prediction: Towards an instance aware problem solver. In: Technical Report: MSR-TR-2005125, Microsoft Research, WA. (2005)

17. Li, C.M., Anbulagan: Look-ahead versus look-back for satisfiability problems. In: Proceedings of 3rd CP. (1997) 341-355

18. Quine, W.V.: A way to simplify truth functions. American Mathematical Monthly 62 (1955) 627-631

19. Davis, M., Putnam, H.: A computing procedure for quantification theory. Journal of the ACM 7 (1960) 201-215

20. Robinson, J.A.: A machine-oriented logic based on the resolution principle. Journal of the ACM 12 (1965) 23-41 